University of Nebraska - Lincoln

DigitalCommons@University of Nebraska - Lincoln

2012

\title{
Enhancement of Thermopower of TAGS-85 High-Performance Thermoelectric Material by Doping with the Rare Earth Dy
}

\author{
E. M. Levin \\ lowa State University, levin@iastate.edu \\ S. L. Bud'ko \\ lowa State University, budko@ameslab.gov \\ K. Schmidt-Rohr \\ lowa State University, srohr@iastate.edu
}

Follow this and additional works at: https://digitalcommons.unl.edu/usdoepub

Part of the Bioresource and Agricultural Engineering Commons

Levin, E. M.; Bud'ko, S. L.; and Schmidt-Rohr, K., "Enhancement of Thermopower of TAGS-85 HighPerformance Thermoelectric Material by Doping with the Rare Earth Dy" (2012). US Department of Energy Publications. 334.

https://digitalcommons.unl.edu/usdoepub/334

This Article is brought to you for free and open access by the U.S. Department of Energy at DigitalCommons@University of Nebraska - Lincoln. It has been accepted for inclusion in US Department of Energy Publications by an authorized administrator of DigitalCommons@University of Nebraska - Lincoln. 


\title{
Enhancement of Thermopower of TAGS-85 High-Performance Thermoelectric Material by Doping with the Rare Earth Dy
}

\author{
E. M. Levin,* S. L. Bud'ko, and K. Schmidt-Rohr
}

Enhancement of thermopower is achieved by doping the narrow-band semiconductor $\mathrm{Ag}_{6.52} \mathrm{Sb}_{6.52} \mathrm{Ge}_{36.96} \mathrm{Te}_{50}$ (acronym TACS-85), one of the best $p$-type thermoelectric materials, with 1 or $2 \%$ of the rare earth dysprosium (Dy). Evidence for the incorporation of Dy into the lattice is provided by $X$-ray diffraction and increased orientation-dependent local fields detected by ${ }^{125} \mathrm{Te}$ NMR spectroscopy. Since Dy has a stable electronic configuration, the enhancement cannot be attributed to 4 -electron states formed near the Fermi level. It is likely that the enhancement is due to a small reduction in the carrier concentration, detected by ${ }^{125} \mathrm{Te}$ NMR spectroscopy, but mostly due to energy filtering of the carriers by potential barriers formed in the lattice by Dy, which has large both atomic size and localized magnetic moment. The interplay between the thermopower, the electrical resistivity, and the thermal conductivity of TACS-85 doped with Dy results in an enhancement of the power factor $(P F)$ and the thermoelectric figure of merit $(Z T)$ at $730 \mathrm{~K}$, from $P F=28 \mu \mathrm{W} \mathrm{cm}-1 \mathrm{~K}^{-2}$ and $Z T \leq 1.3$ in TACS -85 to $P F=35 \mu \mathrm{W} \mathrm{cm}{ }^{-1} \mathrm{~K}^{-2}$ and $Z T \geq 1.5$ in TACS- 85 doped with 1 or $2 \%$ Dy for Ge. This makes TACS- 85 doped with Dy a promising material for thermoelectric power generation.

conversion. $^{[1-3]} \quad$ Thermoelectric power generation is a promising technology for mitigating the effects of the developing fossil-fuel shortage: thermoelectric materials can utilize waste heat at power plants, in automobiles, and in other heat engines. A recent analysis has shown that thermoelectric materials can also be used in solar energy conversion as an alternative to photovoltaic systems. ${ }^{[4,5]}$ For all these applications, one needs new efficient thermoelectric materials with a figure of merit $Z T \geq 2$, which is higher than has been reproducibly achieved so far.

Thermoelectric phenomena have been studied intensively in various materials..$^{[1-3]}$ However, in spite of intensive research, the well known GeTe-, PbTe-, and $\mathrm{Bi}_{2} \mathrm{Te}_{3}$-based systems are still among the best thermoelectric materials. ${ }^{[2]}$ The enhancement of the thermoelectric properties of tellurides can be achieved by doping known materials with atoms of

\section{Introduction}

The direct conversion of heat to electricity using the Seebeck effect, or of electricity to heat removal using the Peltier effect, are fundamentally interesting physical phenomena, and simultaneously attractive and elegant processes for energy

Dr. E. M. Levin, Dr. S. L. Bud'ko, Dr. K. Schmidt-Rohr Division of Materials Sciences and Engineering Ames Laboratory US DOE

lowa State University

Ames, IA 50011-3020, USA

E-mail: levin@iastate.edu

Dr. E. M. Levin, Dr. S. L. Bud'ko

Department of Physics and Astronomy

lowa State University

Ames, IA 50011-3160, USA

Dr. K. Schmidt-Rohr

Department of Chemistry

lowa State University

Ames, IA 50011-3111, USA

DOI: $10.1002 /$ adfm.201103049 relatively large or small atomic sizes, and with or without a localized magnetic moment. It is clear that not only reduction in the thermal conductivity, $\kappa$, but also an enhancement of the power factor, $P F=S^{2} / \rho$, where $S$ is the Seebeck coefficient (thermopower) and $\rho$ the electrical resistivity, must be major goals in order to obtain better thermoelectric materials. Such a modification can achieved using a combination of several dopants; for instance, it was recently reported by Pei et al.[6] that simultaneous doping of $\mathrm{PbTe}$ with $\mathrm{Na}$ and Se results in the enhancement of the thermoelectric properties: this work demonstrates that the well-known semiconductor PbTe can still be used as a matrix in designing high-performance thermoelectric materials. We show here that this is similarly true for the GeTe narrow-band semiconductor.

The thermopower is the most important parameter contributing to the power factor, $P F$, which can be regarded as the electrical part of the thermoelectric figure of merit, $Z T=$ $P F T / \kappa$. In narrow-band semiconductors, the thermopower can be changed via different mechanisms including: i) a change in charge-carrier concentration; ${ }^{[7]}$ ii) a modification of the density of electron states (DOS) near the Fermi level; ${ }^{[8,9]}$ and iii) energy filtering. ${ }^{[1,9-12]}$ The latter explains the thermopower enhancement by differential scattering of high- and 
low-energy carriers by potential barriers formed at the interface between the matrix and various inclusions on the microscale, ${ }^{[1,12]}$ or by defects on the atomic scale. ${ }^{[9]}$ Energy filtering generally is most efficient at low temperatures, but can also be valuable at temperatures of about $600-700 \mathrm{~K} .{ }^{[12]}$ We suggest that energy filtering can occur in materials with strong local lattice distortions (imperfections) formed by dopant atoms with much larger or smaller atomic sizes, compared with the replaced atoms of the matrix. In addition, atoms with large localized magnetic moments distributed in the lattice serve as scattering centers and can also be associated with local lattice imperfections.

The majority of the atoms of transition elements, lanthanides, and actinides carry a magnetic moment due to unpaired $3 d$-, $4 f$-, and $5 f$-electrons, respectively, but only lanthanides may possess large and strongly localized magnetic moments; ${ }^{[13]}$ the exceptions are the "non-magnetic" La and $\mathrm{Lu}$, and $\mathrm{Ce}, \mathrm{Eu}$, and $\mathrm{Yb}$, which have unstable $4 f$-shells. ${ }^{[14]}$ Lanthanides as dopants can produce various effects in the lattice: i) due to their large size, rare-earth atoms can result in local lattice distortions; note that the atomic size of rareearth atoms decreases from La (atomic radius $r_{\mathrm{a}}=0.274 \mathrm{~nm}$ ) to $\mathrm{Lu}\left(r_{\mathrm{a}}=0.225 \mathrm{~nm}\right)$, which is still larger than that of $\mathrm{Ge}\left(r_{\mathrm{a}}=\right.$ $0.152 \mathrm{~nm})$ and $\left.\mathrm{Te}\left(r_{\mathrm{a}}=0.142 \mathrm{~nm}\right) ;{ }^{[15]} \mathrm{ii}\right)$ rare earths may have vanishing (e.g., $\mathrm{La}$ and $\mathrm{Lu}$ ), small (e.g., $\mathrm{Pr}, \mathrm{Nd}$, and $\mathrm{Er}$ ), or large (e.g., Gd, Dy, and $\mathrm{Tb}^{[13]}$ ) localized magnetic moments; iii) some of the rare earths (e.g., $\mathrm{Ce}, \mathrm{Eu}$, and $\mathrm{Yb}$ ) may have unstable electronic configurations and form resonance $4 f$-electron states near the Fermi level; ${ }^{[14]}$ and iv) rare earths can form local bonds and affect the charge-carrier concentration. Note that due to the localized magnetic moments, additional interesting phenomena may occur (e.g., the spin Seebeck effect). ${ }^{[16,17]}$

A well-known group of high-performance thermoelectric materials is GeTe alloyed with $\mathrm{Ag}$ and $\mathrm{Sb}$ on the $\mathrm{Ge}$ sublattice. ${ }^{[18]}$ Commonly, their composition is shown as $(\mathrm{GeTe})_{x}\left(\mathrm{AgSbTe}_{2}\right)_{100-x}$ with $x=75,80,85$, and 90 , or by the acronym "TAGS- $x$ ". The material with $x=85$ (TAGS-85), which can be shown in atomic percent as $\mathrm{Ag}_{6.52} \mathrm{Sb}_{6.52} \mathrm{Ge}_{36.96} \mathrm{Te}_{50}$, is one of the best thermoelectric materials known and has been used for various power generation applications. ${ }^{[19]}$ Recently, we showed ${ }^{[20]}$ that doping of $\mathrm{Ag}_{6.52} \mathrm{Sb}_{6.52} \mathrm{Ge}_{36.96} \mathrm{Te}_{50}$ (TAGS-85) with $\mathrm{Ce}$ or $\mathrm{Yb}$ for $\mathrm{Te}$ enhances the thermoelectric properties. In order to better understand the effect of the doping of $\mathrm{Ag}_{6.52} \mathrm{Sb}_{6.52} \mathrm{Ge}_{36.96} \mathrm{Te}_{50}$ with lanthanides that possess a stable electron configuration and a large localized magnetic moment, we have now studied TAGS-85 where the Ge or Te atoms are replaced by dysprosium, Dy (Dy for Ge or Dy for $\mathrm{Te}$ in the following). For alloys, $\mathrm{Ag}_{6.52} \mathrm{Sb}_{6.52} \mathrm{Ge}_{36.96} \mathrm{Te}_{50}$ (TAGS85), $\mathrm{Ag}_{6.52} \mathrm{Sb}_{6.52} \mathrm{Ge}_{35.96} \mathrm{Te}_{50} \mathrm{Dy}_{1}$ (TAGS-85 + 1\% Dy for Ge), $\mathrm{Ag}_{6.52} \mathrm{Sb}_{6.52} \mathrm{Ge}_{34.96} \mathrm{Te}_{50} \mathrm{Dy}_{2}$ (TAGS-85 + 2\% Dy for $\mathrm{Ge}$ ), and $\mathrm{Ag}_{6.52} \mathrm{Sb}_{6.52} \mathrm{Ge}_{36.96} \mathrm{Te}_{49} \mathrm{Dy}_{1}$ (TAGS-85 + 1\% Dy for Te), have been synthesized and studied by X-ray diffraction (XRD), ${ }^{125} \mathrm{Te}$ NMR spectroscopy, both at $300 \mathrm{~K}$, and by measurements of the thermopower, electrical resistivity, and thermal conductivity in the temperature range from 305 to $750 \mathrm{~K}$. In addition, the dc magnetization was measured from 1.8 to $350 \mathrm{~K}$ in an applied magnetic field up to $55 \mathrm{kOe}$.

\section{Results and Discussion}

\subsection{X-Ray Diffraction}

Figure 1 shows X-ray diffraction (XRD) patterns of TAGS-85, TAGS-85 + 1\% Dy for Ge, TAGS-85 + 2\% Dy for Ge, and TAGS-85 $+1 \%$ Dy for Te. The XRD showed that the Dy-doped materials contain mostly one phase and have a rhombohedral structure similar to the parent TAGS-85 $\left(\mathrm{Ag}_{6.52} \mathrm{Sb}_{6.52} \mathrm{Ge}_{36.96} \mathrm{Te}_{50}\right) \cdot{ }^{[21]}$ The inset in Figure 1 shows an expanded view of the (202) peak at $2 \Theta \approx 30^{\circ}$ for all four samples; the shift of the (202) peak toward smaller angles is evidence that the large Dy atoms were embedded, at least partially, in the lattice, at both $\mathrm{Ge}$ and Te sites. A similar shift was observed for the (024) and (220) peaks at $2 \Theta \approx 42^{\circ}$ and $\approx 43.5^{\circ}$, respectively. The effect of the replacement increased with the Dy content and was larger when Dy replaced Ge.

\subsection{Magnetization}

Figure 2 shows plots of: a) mass magnetization, $M$, vs. magnetic field, $H$; and b) the $M / H$ ratio vs. the temperature of TAGS-85, TAGS-85 + 1\% Dy for Ge, TAGS-85+ 2\% Dy for Ge, and TAGS-85 $+1 \%$ Dy for Te. At $300 \mathrm{~K}$, TAGS-85 exhibited negative (diamagnetic) magnetization, $M=-2.95 \times 10^{-2} \mathrm{emu} \mathrm{g}^{-1}$ when measured in an $H=50$ kOe magnetic field (Figure 2a); the diamagnetic susceptibility is $\chi_{\text {dia }}=M / H \approx-6 \times 10^{-7} \mathrm{emu} \mathrm{g}^{-1} \mathrm{Oe}^{-1}$. All of the samples doped with Dy showed positive (paramagnetic) magnetization. At $1.8 \mathrm{~K}$ (see inset in Figure 2 a), the magnetization exhibited a saturated behavior, which can be observed for some paramagnetic materials containing localized magnetic moments and can be described by the Brillouin function. ${ }^{[13,22]}$ The temperature dependence of the ratio $M / H$, which in diamagnetic and paramagnetic materials represents the magnetic susceptibility, $\chi$, showed behavior typical of paramagnetic systems (Figure $2 \mathrm{~b}$ ).

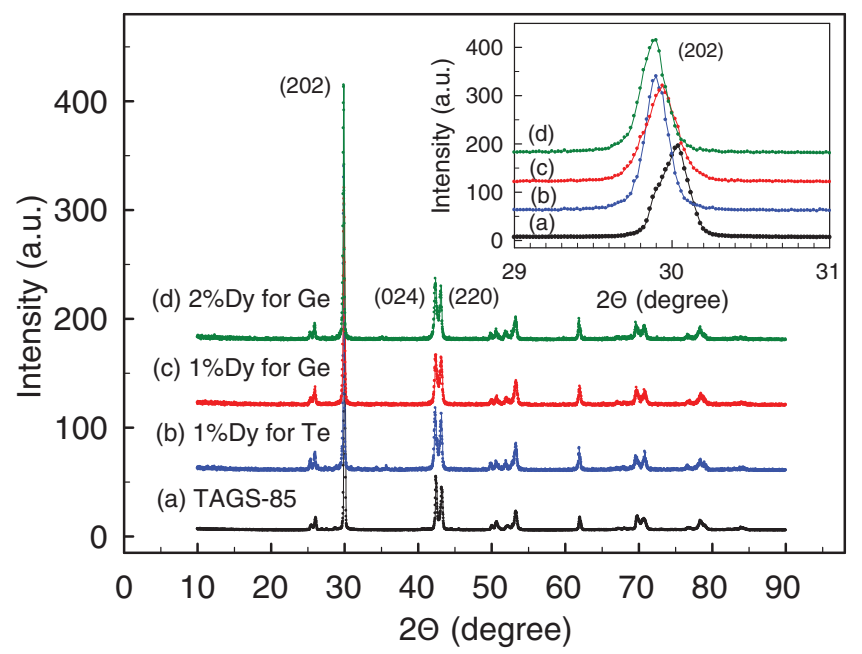

Figure 1. X-ray diffraction of a) $\mathrm{Ag}_{6.52} \mathrm{Sb}_{6.52} \mathrm{Ge}_{36.96} \mathrm{Te}_{50}$ (TAGS85), b) $\mathrm{Ag}_{6.52} \mathrm{Sb}_{6.52} \mathrm{Ge}_{36.96} \mathrm{Te}_{49} \mathrm{Dy}_{1}$ (TAGS-85 + 1\% Dy for Te), c) $\mathrm{Ag}_{6.52} \mathrm{Sb}_{6.52} \mathrm{Ce}_{35.96} \mathrm{Te}_{50} \mathrm{Dy} y_{1}$ (TACS-85 $+1 \% \mathrm{Dy}$ for Ge), and d) $\mathrm{Ag}_{6.52} \mathrm{Sb}_{6.52} \mathrm{Ge}_{34.96} \mathrm{Te}_{50} \mathrm{Dy}_{2}$ (TACS-85 + 2\% Dy for $\mathrm{Ge}$ ). The inset shows an expanded view of the (202) peak. 

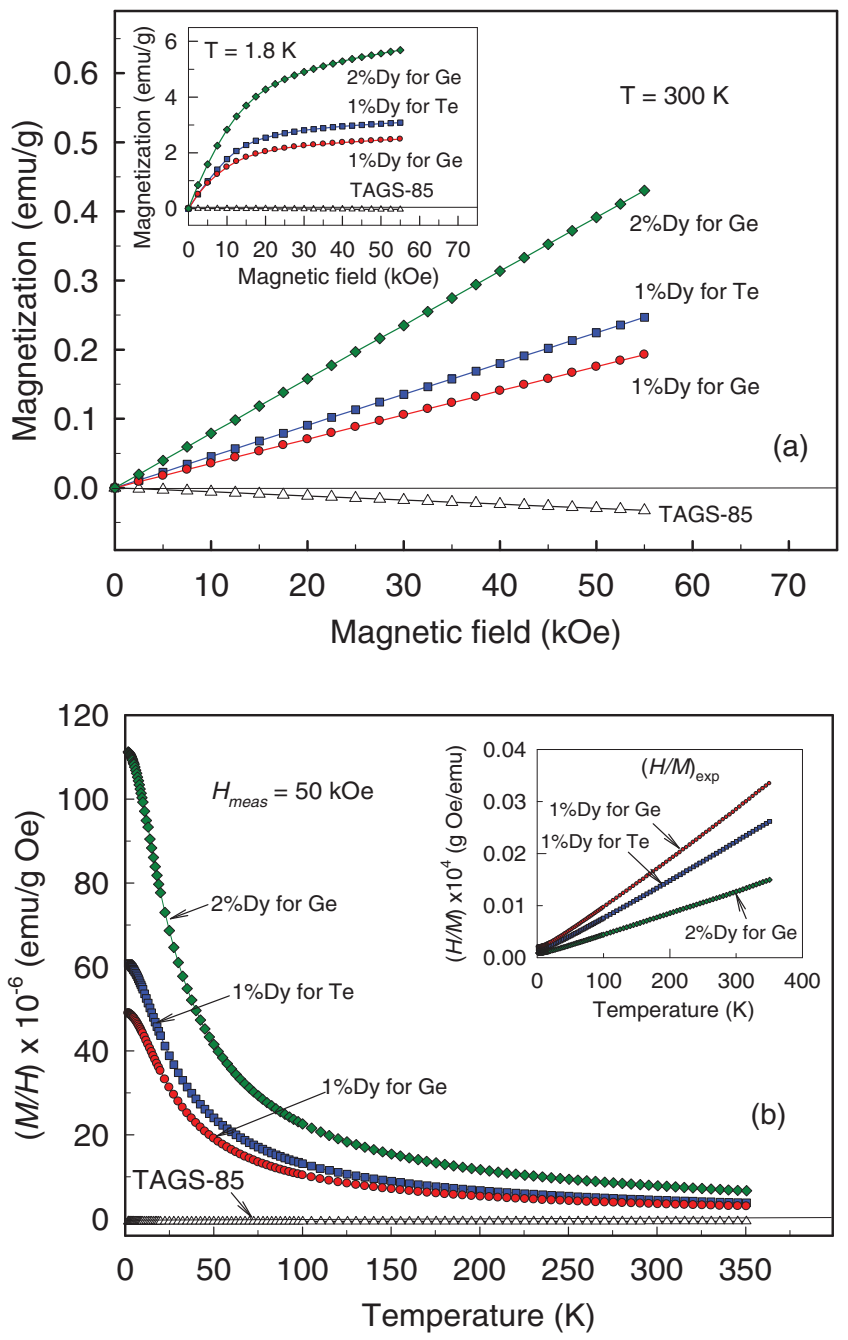

Figure 2. a) Magnetization, $M$, measured at $300 \mathrm{~K}$, and b) temperature dependencies of the measured $(M / H)_{\exp }$ ratio of $\mathrm{Ag}_{6.52} \mathrm{Sb}_{6.52} \mathrm{Ce}_{36.96} \mathrm{Te}_{50}$ (TAGS-85), $\mathrm{Ag}_{6.52} \mathrm{Sb}_{6.52} \mathrm{Ce}_{36.96} \mathrm{Te}_{49} \mathrm{Dy}_{1}$ (TAGS-85 + $1 \%$ Dy for Te), $\mathrm{Ag}_{65} \mathrm{Sb}_{6.52} \mathrm{Ce}_{35}{ }_{96} \mathrm{Te}_{50} \mathrm{Dy}$ (TAGS-85 + 1\% Dy for Ge), and $\mathrm{Ag}_{6.52} \mathrm{Sb}_{6.52} \mathrm{Ce}_{34.96} \mathrm{Te}_{50} \mathrm{Dy}{ }_{2}$ (TACS-85 + 2\% Dy for Ge). The inset in (a) shows the magnetization measured at $1.8 \mathrm{~K}$; the inset in (b) shows the $H / M$ ratio vs. temperature for all three samples doped with Dy.

The inset in Figure $2 \mathrm{~b}$ shows the inverse $H / M=1 / \chi$ ratio, which fits the Curie law and indicates that Dy ions formed a system of non-interacting localized magnetic moments. According to the Curie law, the paramagnetic susceptibility increases with decreasing temperature and can be expressed as: ${ }^{[13]}$

$\chi_{\mathrm{par}(\mathrm{mol})}=\left(N p_{\text {eff }}^{2}\right) / 3 k T=C / T$

where $\chi_{\mathrm{par}(\mathrm{mol})}=\left(M_{\mathrm{m}} / H\right)_{\mathrm{par}}=\chi_{\mathrm{par}} m_{\mathrm{mol}}$ is the molar magnetic susceptibility, $m_{\text {mol }}$ is the molar mass of the formula unit of the substance, $N$ is the Avogadro number, $k$ is the Boltzmann constant, and $p_{\text {eff }}$ is the effective molar magnetic moment. Using the magnetic susceptibility at $1.8 \mathrm{~K}$, we determined the Curie constant, $C=$ $\chi_{\text {par }} T$, for $\mathrm{Ag}_{6.52} \mathrm{Sb}_{6.52} \mathrm{Ge}_{36.96} \mathrm{Te}_{49} \mathrm{Dy}_{1}$ (TAGS-85 + 1\% Dy for Te) and $\mathrm{Ag}_{6.52} \mathrm{Sb}_{6.52} \mathrm{Ge}_{35.96} \mathrm{Te}_{50} \mathrm{Dy}_{1}$ (TAGS-85 + 1\% Dy for Ge) to be
$11.0 \times 10^{-5}$ and $8.8 \times 10^{-5} \mathrm{~K} \mathrm{emu} \mathrm{g}^{-1} \mathrm{Oe}^{-1}$, respectively. The effective magnetic moments per Dy atom calculated using Equation 1 are 10.7 and $9.5 \mu_{\mathrm{B}}$, respectively, whereas the theoretical value for a Dy atom is $10.64 \mu_{\mathrm{B}} \cdot{ }^{[13]}$ For $\mathrm{Ag}_{6.52} \mathrm{Sb}_{6.52} \mathrm{Ge}_{34.96} \mathrm{Te}_{50.00} \mathrm{Dy}_{2.00}$ (TAGS- $85+2 \%$ Dy for Ge), we found $p_{\text {eff }}=10.1 \mu_{\mathrm{B}}$. The values of the effective magnetic moments being smaller than the theoretical one can be attributed to a slightly lower Dy concentration in the lattice than expected for the nominal composition.

\section{3. ${ }^{125}$ Te NMR Spectra and Spin-Lattice Relaxation}

Figure 3 shows ${ }^{125}$ Te NMR data for TAGS-85 without and with Dy doping. The spectra (Figure 3a) exhibit a systematic shift of
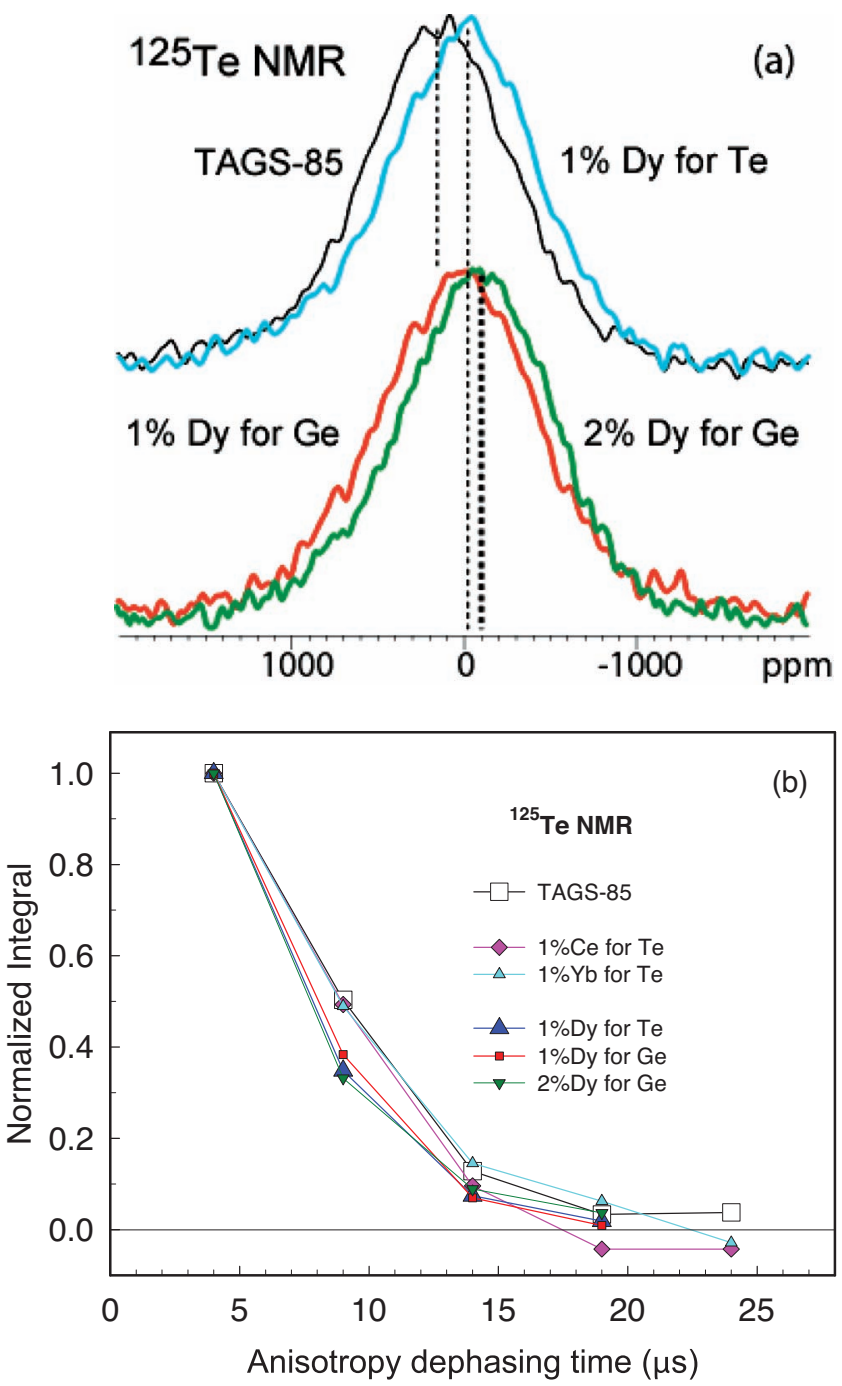

Figure 3. a) ${ }^{125} \mathrm{Te}$ NMR spectra of TAGS-85, TAGS-85 + 1\% Dy for Te, TACS- $85+1 \%$ Dy for Ge, and TACS- 85 + 2\% Dy for Ge, and b) intensity of the ${ }^{125} \mathrm{Te}$ NMR signal as a function of recoupling time in an experiment probing orientation-dependent interactions of the ${ }^{125} \mathrm{Te}$ nuclei for TACS-85 doped with Dy. Data for TAGS-85 doped with Ce or Yb for Te ${ }^{[20]}$ are shown for comparison. Signal reduction due to regular $T_{2}$ relaxation during the short dephasing period $(<50 \mu \mathrm{s})$ is negligible $(<5 \%)$. 
the peak maximum to the right with Dy concentration, indicative of a smaller Knight shift. The spin-lattice relaxation time in TAGS-85 $+2 \%$ Dy for Ge was $T_{1}=3.8 \mathrm{~ms}$, while $T_{1}$ for TAGS-85 was $3.1 \mathrm{~ms}$. Figure $3 \mathrm{~b}$ displays a plot of the ${ }^{125}$ Te NMR signal intensities of neat and doped TAGS as a function of recoupling time in an experiment that reflects the orientation-dependent local magnetic fields probed by the ${ }^{125} \mathrm{Te}$ nuclei. ${ }^{[23]}$ Data for TAGS-85 doped with Ce or $\mathrm{Yb}^{[20]}$ are shown for comparison. In neat TAGS, the decay mostly reflects the chemical-shift anisotropy due to deviations from local cubic symmetry caused by the rhombohedral distortion, alloying and vacancies.

The dephasing for TAGS doped with $\mathrm{Ce}$ or $\mathrm{Yb}$ was very similar, while faster dephasing was observed for the Dy-doped TAGS samples. Simulations have shown that the increased dephasing rate corresponds to an interaction of approximately $8 \mathrm{kHz} .{ }^{[23]}$ We attribute the faster dephasing to magnetic-dipole couplings between the magnetic moment of Dy and the ${ }^{125} \mathrm{Te}$ nuclear magnetic moments. Given that the dipolar field drops off with the inverse cube of the distance between the ion and the nucleus, this is evidence that Dy is incorporated into the TAGS-85 lattice.

\subsection{Seebeck Coefficient, Electrical Resistivity, and Power Factor}

Figure 4 shows the dependence of the absolute Seebeck coefficient, $S$, measured at average temperatures of 305 and 700 $\mathrm{K}$ for $\mathrm{Ag}_{6.52} \mathrm{Sb}_{6.52} \mathrm{Ge}_{35.96} \mathrm{Te}_{50} \mathrm{Dy}_{1}$ (TAGS-85 + 1\% Gd for Ge), $\mathrm{Ag}_{6.52} \mathrm{Sb}_{6.52} \mathrm{Ge}_{34.96} \mathrm{Te}_{50} \mathrm{Dy}_{2}$ (TAGS-85 + $2 \% \mathrm{Gd}$ for $\mathrm{Ge}$ ), and $\mathrm{Ag}_{6.52} \mathrm{Sb}_{6.52} \mathrm{Ge}_{36.96} \mathrm{Te}_{49} \mathrm{Dy}_{1}$ (TAGS-85 + 1\% Dy for Te), vs. the distance from the top of the ingot. Measurements were made for 6 samples sectioned at the top ( 3 samples) and at the bottom (3 samples) of each ingot for all three materials studied.

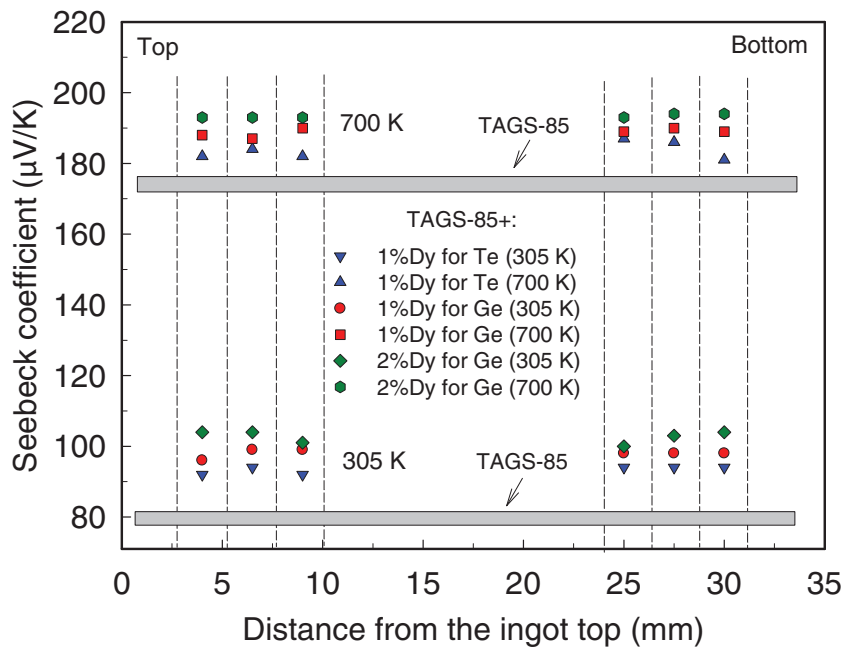

Figure 4. Variation of the absolute Seebeck coefficient of TAGS-85 doped with 1 or $2 \%$ Dy for Ge or with $1 \%$ Dy for Te vs. the distance from the ingot top at 305 and $700 \mathrm{~K}$. The two horizontal gray bars show the range of the absolute Seebeck coefficient of two $\mathrm{Ag}_{6.52} \mathrm{Sb}_{6.52} \mathrm{Ge}_{36.96} \mathrm{Te}_{50}$ (TAGS $85)$ samples, at 305 and $700 \mathrm{~K}$. The vertical, dashed lines indicate the cuts of the ingot made using a diamond saw; the thickness of each slice (i.e., thickness of each sample) was $\approx 2 \mathrm{~mm}$.
The absolute Seebeck coefficients of $\mathrm{Ag}_{6.52} \mathrm{Sb}_{6.52} \mathrm{Ge}_{36.96} \mathrm{Te}_{50}$ (TAGS-85) at the respective temperatures are shown by the two horizontal bars, which reflect the values of thermopower of the two different TAGS-85 samples: the lower edge of the bars marks $S$ for TAGS-85 \#1 (studied by Levin et al. ${ }^{[20]}$ ), and the upper edge marks $S$ for TAGS-85 \#2 (synthesized for the current study). The plot in Figure 4 exhibits several interesting features: the Seebeck coefficient of TAGS-85 doped with Dy for $\mathrm{Ge}$ is nearly constant along the ingot and is definitely larger than that of both the TAGS-85 \#1 and \#2 samples. In addition, the Seebeck coefficient of the samples where Dy replaced Ge is larger than that for Dy replacing Te. A larger enhancement of the absolute thermopower is observed for TAGS-85 doped with $2 \%$ Dy for Ge.

Figure 5 shows the temperature dependencies of: a) the absolute Seebeck coefficient, $S$; and b) the electrical resistivity,
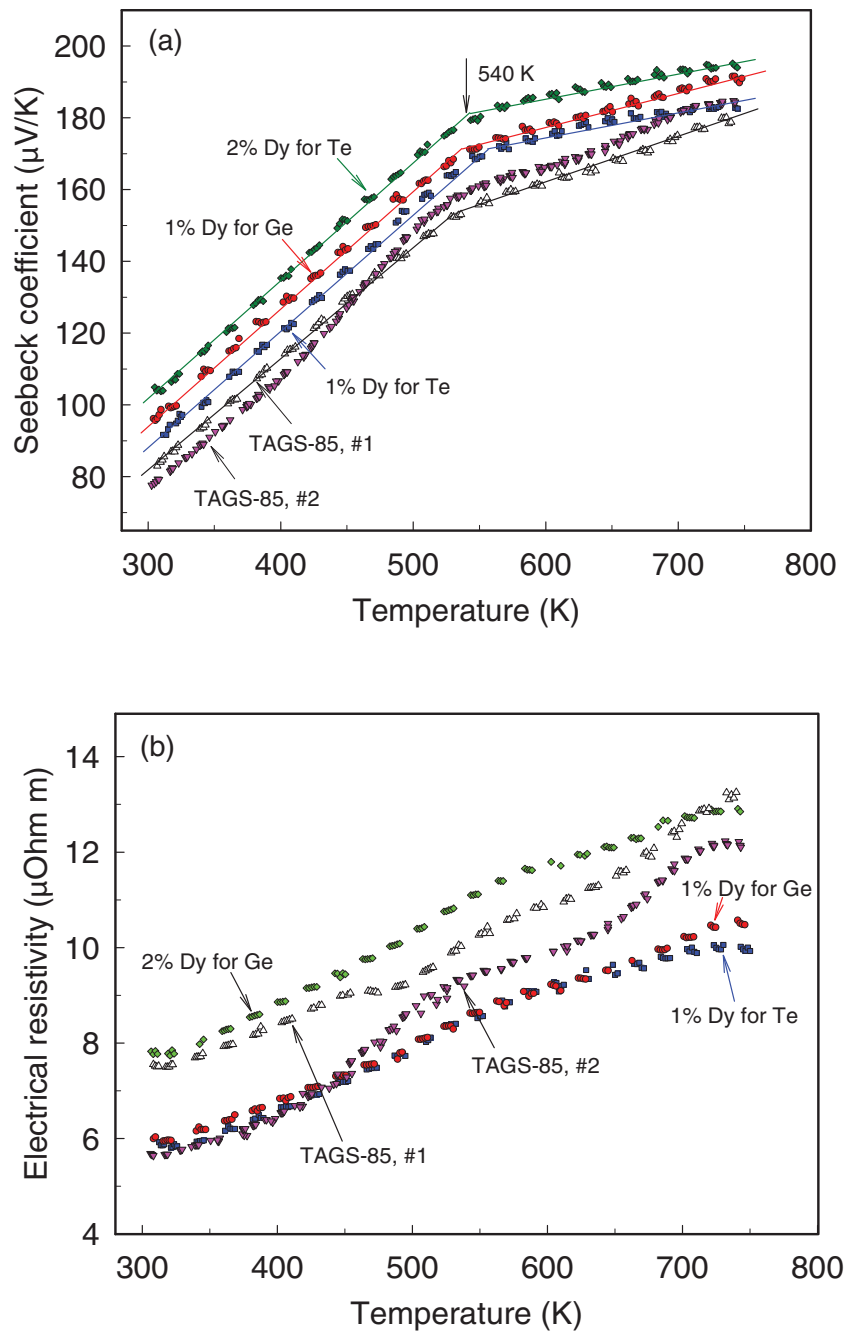

Figure 5. a) Temperature dependences of the absolute Seebeck coefficient, $S$, and $b$ ) the electrical resistivity, $\rho$, of the two samples of $\mathrm{Ag}_{6.52} \mathrm{Sb}_{6.52} \mathrm{Ce}_{36.96} \mathrm{Te}_{50}$ (TAGS-85), $\mathrm{Ag}_{6.52} \mathrm{Sb}_{6.52} \mathrm{Ge}_{36.96} \mathrm{Te}_{49} \mathrm{Dy}$ (TACS-85 + $1 \%$ Dy for Te), $\mathrm{Ag}_{6.52} \mathrm{Sb}_{6.52} \mathrm{Ge}_{35.96} \mathrm{Te}_{50} \mathrm{Dy}{ }_{1}$ (TACS-85 + 1\% Dy for Ge), and $\mathrm{Ag}_{6.52} \mathrm{Sb}_{6.52} \mathrm{Ce}_{34.96} \mathrm{Te}_{50} \mathrm{Dy} 2$ (TACS-85 + 2\% Dy for $\mathrm{Ge}$ ) extracted from the tops of the ingots. 
$\rho$, of four samples extracted from the tops of the ingots: $\mathrm{Ag}_{6.52} \mathrm{Sb}_{6.52} \mathrm{Ge}_{36.96} \mathrm{Te}_{50} \quad$ (TAGS-85), $\quad \mathrm{Ag}_{6.52} \mathrm{Sb}_{6.52} \mathrm{Ge}_{35.96} \mathrm{Te}_{50} \mathrm{Dy}_{1}$ (TAGS-85 + 1\% Dy for Ge), $\mathrm{Ag}_{6.52} \mathrm{Sb}_{6.52} \mathrm{Ge}_{34.96} \mathrm{Te}_{50} \mathrm{Dy}_{2}$ (TAGS-85 + $2 \%$ Dy for $\mathrm{Ge}$ ), and $\mathrm{Ag}_{6.52} \mathrm{Sb}_{6.52} \mathrm{Ge}_{36.96} \mathrm{Te}_{49} \mathrm{Dy}_{1}$ (TAGS-85 + 1\% Dy for Te). The absolute Seebeck coefficients of the TAGS-85 samples doped with Dy, $S_{730 \mathrm{~K}}=+188 \mu \mathrm{V} \mathrm{K} \mathrm{K}^{-1}$ and $S_{730 \mathrm{~K}}=$ $+195 \mu \mathrm{V} \mathrm{K} \mathrm{K}^{-1}$ when doped with 1 or $2 \%$ Dy, respectively, were higher than the values for neat TAGS-85, $+172 \mu \mathrm{V} \mathrm{K} \mathrm{K}^{-1}$ and $+176 \mu \mathrm{V} \mathrm{K}^{-1}$, for samples \#1 and \#2, respectively. The temperature dependence of the thermopower of all of the samples shows two regions with a nearly linear increase, but different slopes. The slopes in the temperature range of (i) 305-520 K are similar, $0.36 \mu \mathrm{V} \mathrm{K}^{-2}$, whereas those in the range of (ii) 540 $760 \mathrm{~K}$ depend on the composition and varied between 0.13 and $0.08 \mu \mathrm{V} \mathrm{K} \mathrm{K}^{-2}$ for TAGS-85 and TAGS-85 + 2\% Dy for Ge, respectively. At $300 \mathrm{~K}$, TAGS-85 has a rhombohedral structure with an angle of $88^{\circ}$, which gradually increases with temperature and reaches $90^{\circ}$ at $\approx 510 \mathrm{~K} ;{ }^{[21]}$ above this temperature the crystal lattice of TAGS- 85 has cubic symmetry. The temperature of the change in the thermopower slope, $\approx 525 \mathrm{~K}$, was close to the region where the rhombohedral distortion disappeared. Hence, the large thermopower slopes in temperature range (i) can be associated with the rhombohedral structure, while the smaller ones in the range (ii) can be associated with the cubic structure.

The temperature dependence of the electrical resistivity is typical of degenerate semiconductors where the increase of the electrical resistivity with temperature is mostly associated with an increase in carrier scattering by phonons. Some contribution to the electrical resistivity may arise due to porosity and microcracks, so we could not easily distinguish the contributions arising from carrier concentration, mobility, and point defects, and due to porosity and microcracks. Generally, TAGS-85 samples doped with Dy had less visually observable porosity than neat TAGS-85. Furthermore, in TAGS-85 doped with Dy, the porosity at the top of the ingot was less than at the bottom. The lowest values of the electrical resistivity of the samples with 1 or $2 \%$ Dy for Ge measured at $730 \mathrm{~K}, 10 \mu \Omega \mathrm{m}$, and $13 \mu \Omega \mathrm{m}$, respectively, were found for the samples extracted from the tops of all the ingots.

Figure 6 shows the power factor, $P F=S^{2} / \rho$, calculated for the same samples. PF reflects the electrical part of the thermoelectric figure of merit but its value strongly depends not only on the thermopower, which is insensitive to porosity and microcracks in the material, but also on the measured value of the electrical resistivity, which is sensitive to these microscopic defects. The power factor of $\mathrm{Ag}_{6.52} \mathrm{Sb}_{6.52} \mathrm{Ge}_{35.96} \mathrm{Te}_{50} \mathrm{Dy}_{1}$ (TAGS-85 doped with $1 \%$ Dy for Ge) is $P F_{730 \mathrm{~K}}=35 \mu \mathrm{W} \mathrm{cm}{ }^{-1} \mathrm{~K}^{-2}$, while that of TAGS-85 \#1 and TAGS-85 \#2 are 24 and $28 \mu \mathrm{W} \mathrm{cm}{ }^{-1} \mathrm{~K}^{-2}$, respectively. Although the absolute Seebeck coefficient of $\mathrm{Ag}_{6.52} \mathrm{Sb}_{6.52} \mathrm{Ge}_{34.96} \mathrm{Te}_{50} \mathrm{Dy}_{2}$ (TAGS-85 + 2\% Dy for Ge) was even larger than that of TAGS-85 doped with $1 \%$ Dy, the power factor was smaller, $P F_{730 \mathrm{~K}}=$ $29 \mu \mathrm{W} \mathrm{cm}{ }^{-1} \mathrm{~K}^{-2}$, due to the larger electrical resistivity. Note that the power factors of all of the samples studied were nearly constant in the temperature range between $\approx 530 \mathrm{~K}$ and $730 \mathrm{~K}$.

\subsection{Thermal Conductivity and Thermoelectric Figure of Merit}

The temperature dependences of the thermal conductivity and the thermoelectric figure of merit of TAGS-85 without

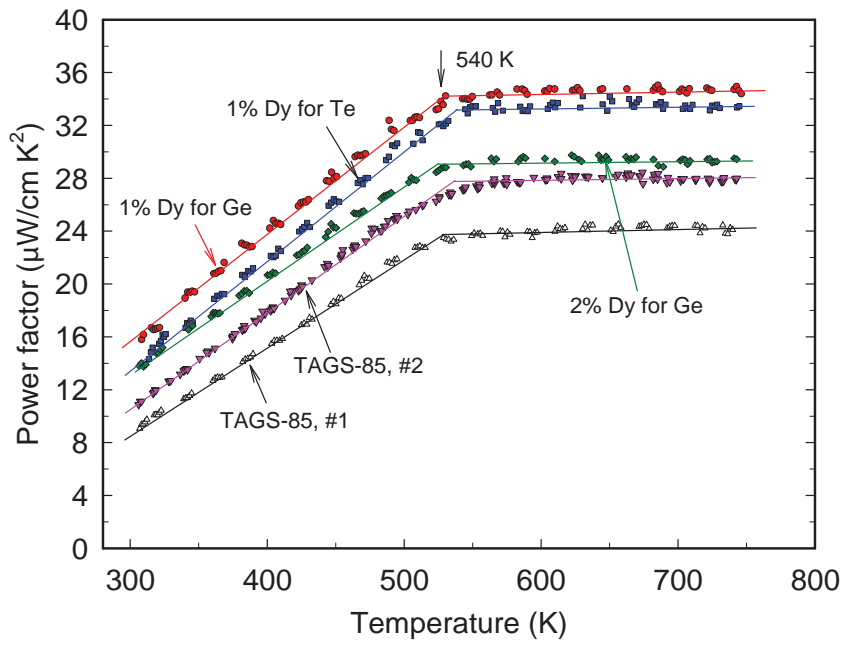

Figure 6. Power factor of the two $\mathrm{Ag}_{6.52} \mathrm{Sb}_{6.52} \mathrm{Ge}_{36.96} \mathrm{Te}_{50}$ (TAGS85) samples, of $\mathrm{Ag}_{6.52} \mathrm{Sb}_{6.52} \mathrm{Ce}_{36.96} \mathrm{Te}_{49} \mathrm{Dy}_{1}$ (TAGS-85 + 1\% Dy for $\mathrm{Te}$ ), of $\mathrm{Ag}_{6.52} \mathrm{Sb}_{6.52} \mathrm{Ce}_{35.96} \mathrm{Te}_{50} \mathrm{Dy}$ (TACS-85 + 1\% Dy for $\mathrm{Ge}$ ), and of $\mathrm{Ag}_{6.52} \mathrm{Sb}_{6.52} \mathrm{Ce}_{34.96} \mathrm{Te}_{50} \mathrm{Dy}_{2}$ (TACS-85 + 2\% Dy for Ge).

and with Dy doping are shown in Figure 7; our data for $\mathrm{GeTe}$ are shown for comparison. At $300 \mathrm{~K}$, the thermal conductivity of TAGS-85 is about 5 times lower than that of $\mathrm{GeTe}$ (Figure 7a) (i.e., simultaneous alloying of $\mathrm{GeTe}$ with $\approx 13 \mathrm{at} \% \mathrm{Ag}$ and $\approx 13 \mathrm{at} \% \mathrm{Sb}$ on the Ge sublattice significantly reduced the thermal conductivity). Note that our data for the thermal diffusivity, $0.5 \mathrm{~cm}^{2} \mathrm{~s}^{-1}$, and the thermal conductivity, $80 \mathrm{~mW} \mathrm{~cm}{ }^{-1} \mathrm{~K}^{-1}$, of GeTe at $300 \mathrm{~K}$ agree well with the literature data. ${ }^{[24-25]}$ Our measurement of the thermal conductivity of TAGS-85 \#2 was $19 \mathrm{~mW} \mathrm{~cm}^{-1} \mathrm{~K}^{-1}$ at $300 \mathrm{~K}$, which is larger than the value of $16 \mathrm{~mW} \mathrm{~cm}^{-1} \mathrm{~K}^{-1}$ reported by Yang et al. ${ }^{[26]}$ and Salvador et al. ${ }^{[27]}$ Note also that TAGS-85 \#1 shows a low thermal conductivity of $16 \mathrm{~mW} \mathrm{~cm}^{-1} \mathrm{~K}^{-1}$ at $300 \mathrm{~K} \cdot{ }^{[20]}$

Doping of TAGS-85 with Dy for Ge reduced the thermal conductivity at $300 \mathrm{~K}$ to 17 and $16 \mathrm{~mW} \mathrm{~cm}^{-1} \mathrm{~K}^{-1}$ for $1 \%$ and $2 \%$ Dy, respectively. However, at $700 \mathrm{~K}$, within the uncertainty of the measurements, the thermal conductivity of TAGS-85 and TAGS-85 doped with Dy samples was similar. The exception is TAGS- $85+2 \%$ Dy, whose thermal conductivity at $700 \mathrm{~K}$ was lower, $\leq 14 \mathrm{~mW} \mathrm{~cm}^{-1} \mathrm{~K}^{-1}$. Figure $7 \mathrm{~b}$ shows the temperature dependences of the thermoelectric figure of merit, $Z T=P F T / \kappa=S^{2} T / \kappa \rho$ for TAGS-85 without and with doping of Dy for Te or Ge. At $\approx 730 \mathrm{~K}$, both of the TAGS-85 samples showed $Z T \leq 1.3$, which is consistent with our value obtained for the TAGS-85 \#1 sample using home-made equipment, ${ }^{[20]}$ and also with the values reported by Yang et al. ${ }^{[26]}$ and Salvador et al., ${ }^{[27]}$ but smaller than the value of $Z T=1.4$ reported by Skrabek and Trimmer. ${ }^{[28]}$ The TAGS-85 sample doped with $1 \%$ Dy for Te showed $Z T \leq 1.5$ at $730 \mathrm{~K}$, whereas $Z T \geq 1.5$ for TAGS-85 doped with 1 or $2 \%$ Dy for Ge. Note that, in spite of the power factor of TAGS-85 doped with $2 \%$ Dy for Ge being smaller than that of TAGS-85 doped with $1 \%$ Dy for $\mathrm{Ge}$, the lower thermal conductivity resulted in a similar, high $Z T$. 

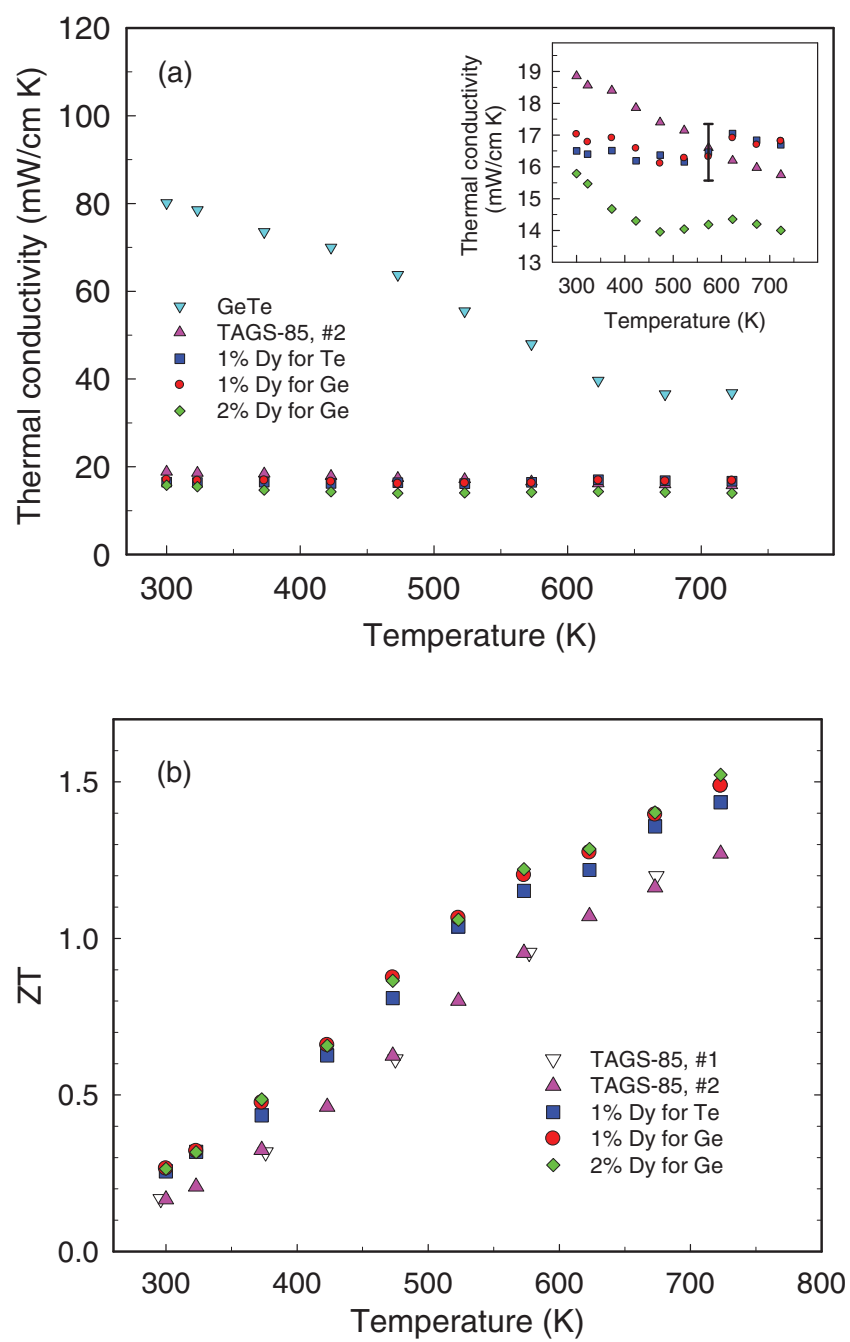

Figure 7. a) Thermal conductivity and b) thermoelectric figure of merit of $\mathrm{Ag}_{6.52} \mathrm{Sb}_{6.52} \mathrm{Ce}_{36.96} \mathrm{Te}_{50}$ (TAGS-85 \#2), $\mathrm{Ag}_{6.52} \mathrm{Sb}_{6.52} \mathrm{Ce}_{36.96} \mathrm{Te}_{49} \mathrm{Dy}_{1}$ (TAGS-85 + $1 \%$ Dy for Te), $\mathrm{Ag}_{6.52} \mathrm{Sb}_{6.52} \mathrm{Ce}_{35.96} \mathrm{Te}_{50} \mathrm{Dy}$ (TACS-85 + 1\% Dy for Ge), and $\mathrm{Ag}_{6.52} \mathrm{Sb}_{6.52} \mathrm{Ge}_{34.96} \mathrm{Te}_{50} \mathrm{Dy}{ }_{2}$ (TAGS-85 + 2\% Dy for Ge). The thermal conductivity of GeTe is shown for reference in (a). The inset in Figure 7 a shows an expanded view of the thermal conductivity.

\subsection{Possible Mechanisms of the Power-Factor Enhancement Due to Doping with Dy}

One of the most important questions is through what mechanisms Dy atoms affect the thermopower of the TAGS-85 thermoelectric material, resulting in the enhancement of the power factor. Elucidation of these mechanisms would provide a better understanding of the effect of rare-earth doping on the thermoelectric properties of complex chalcogenides. Generally, the thermopower can increase due to: i) reduction of the carrier concentration; ii) modification of the DOS near the Fermi level; and/or iii) enhancement of the energy filtering mechanism (see the introduction). The thermal conductivity can decrease due to: i) reduction of the carrier concentration; ii) reduction of the lattice conductivity by changes in the phonon spectra; and iii) possible inclusions. Our data show that doping of GeTe-based complex tellurides with rare earths is an efficient way of modifying both electronic and thermal transport.

Both XRD and ${ }^{125} \mathrm{Te}$ NMR spectroscopy showed that some Dy atoms were incorporated into the lattice. Hence, we can conclude that, in spite of the large atomic radius of Dy $\left(r_{\mathrm{a}}=\right.$ $0.249 \mathrm{~nm})$ compared with that of Ge $\left(r_{\mathrm{a}}=0.152 \mathrm{~nm}\right)$ and Te $\left(r_{\mathrm{a}}=\right.$ $0.142 \mathrm{~nm}$ ), dysprosium may replace Ge and Te or fill vacancies on the Ge sublattice. Figure 8 shows a schematic representation of a magnetic semiconductor such as $\mathrm{Ag}_{\gamma} \mathrm{Sb}_{\gamma} \mathrm{Ge}_{50-2 \gamma-z} \mathrm{Te}_{50} \mathrm{Dy}_{z}$; there should be two main local lattice distortions in this alloy, due to Ge vacancies (lattice shrinks) and Dy doping (lattice expands), and both can contribute to the carrier and phonon scattering (i.e., non-magnetic scattering). Also, due to their large localized magnetic moment Dy atoms can enhance carrier scattering similarly, as observed by Slack and Galginaitis ${ }^{[2]}$ for the narrow-band semiconductor CdTe doped with Mn or Fe.

The temperature dependence of the thermopower of the two TAGS-85 samples is somewhat different from those reported previously. ${ }^{[26-28]}$ At $305 \mathrm{~K}$, our values of the absolute Seebeck coefficient of TAGS-85 \#1 and \#2, $S=+78$ and $+82 \mu \mathrm{V} \mathrm{K}$, respectively, are smaller than those reported by Yang et al. ${ }^{[26]}$ $(S \approx+110 \mu \mathrm{V} \mathrm{K})$ and Salvador et al. ${ }^{[27]}(S=+100 \mu \mathrm{V} \mathrm{K})$. At $730 \mathrm{~K}$, our values for TAGS-85 \#1 and \#2 were $S=+172$ and $+176 \mu \mathrm{V}$ K, respectively. The Seebeck coefficient values reported by Skrabek and Trimmer ${ }^{[28]}$ are larger $(+180$ and $+198 \mu \mathrm{V} \mathrm{K}$ at 600 and $750 \mathrm{~K}$, respectively). The observed differences can be attributed to the conditions and procedures of the measurements, and using the relative instead of the absolute thermopower (see experimental details in Experimental Section).

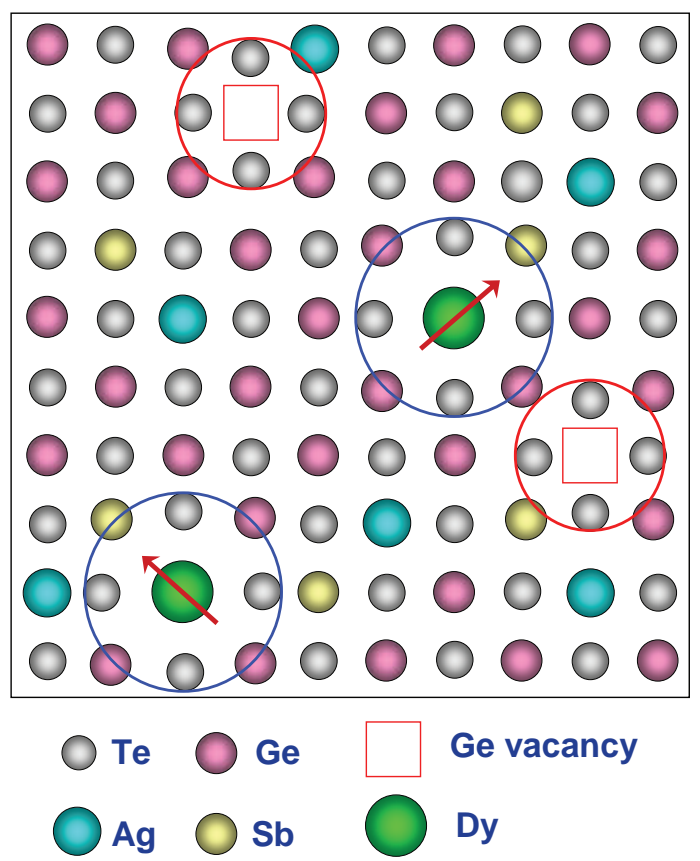

Figure 8. Schematic representation of $\mathrm{Ag}_{y} \mathrm{Sb}_{y} \mathrm{Ge}_{50-2 y-z} \mathrm{Te}_{50} \mathrm{Dy}_{z}$ dilute magnetic semiconductor; local lattice distortions due to Ge vacancies (lattice shrinks) and Dy doping for Ge (lattice expands) are shown by the small red and large blue circles, respectively. The red arrows represent the localized magnetic moments of Dy. 
The literature and our data show that thermoelectric properties of TAGS-85 vary to some extent; this can be explained by variations in the composition and parameters of synthesis, and different contributions from various mechanisms.

The large reduction in the thermal conductivity at $300 \mathrm{~K}$ in TAGS-85, compared with that in GeTe, as well as the decrease in thermal conductivity of TAGS-85 doped with Dy, is mostly due to changes in thermal diffusivity, while the heat capacities and densities of all these materials are very similar. Because our measurements were conducted under the same conditions, and since samples extracted from different locations in the ingot showed similar thermopowers (Figure 4), we can conclude that the doping of TAGS-85 with Dy definitely enhances the thermopower, particularly when Dy atoms replace Ge.

The shift of the ${ }^{125}$ Te NMR frequency observed for TAGS-85 doped with Dy (Figure 3a,b) can be attributed to a slightly reduced Knight shift and, therefore, to a reduction $(\leq 15 \%)$ in carrier concentration, which is insignificant at the level of $\approx 6 \times 10^{20} \mathrm{~cm}^{-3}$. The spin-lattice relaxation, which is driven by the hyperfine coupling of the nuclei to the charge carriers, was slower in TAGS-85 doped with $2 \%$ Dy for Ge, $T_{1}=3.8 \mathrm{~ms}$ than in neat TAGS-85, $T_{1}=3.1 \mathrm{~ms}$, which can be attributed to a slight reduction in the carrier concentration due to Dy doping. Note that ${ }^{125}$ Te NMR spectroscopy (Section 2.3.), as well as the magnetization data (Section 2.2.), in addition to XRD (Section 2.1.), indicate that some amount of Dy atoms is present at the $\mathrm{Ge}$ or $\mathrm{Te}$ sites. The reduction in carrier concentration generally results in a favorable increase of thermopower and also in a partial reduction in thermal conductivity, as indeed observed for TAGS-85 doped with 2\% Dy. However, the moderate change in carrier concentration cannot explain the significant enhancement of thermopower observed in TAGS-85 due to doping with Dy. Hence, we need to consider additional mechanisms of thermopower enhancement (e.g., modification of the DOS or an energy-filtering mechanism).

A large increase of the DOS near the Fermi level, $\varepsilon_{\mathrm{F}}$, due to 4 -electrons is, in principle, possible for $\mathrm{Ce}, \mathrm{Eu}$, and $\mathrm{Yb},{ }^{[14]}$ but not for Dy, because its 4 -electron states are located deeply in the valence band, far from the Fermi level. Ab initio calculations ${ }^{[30]}$ have shown that an increase of the DOS near $\varepsilon_{\mathrm{F}}$ in narrow-gap semiconductors due to $s$ - or $p$-electron states is possible for various impurities: in PbTe, s- dopants (e.g., Zn $\left(3 d^{10} 4 s^{2}\right), \mathrm{Cd}\left(4 d^{10} 5 s^{2}\right)$, and $\left.\mathrm{Hg}\left(5 d^{10} 6 s^{2}\right)\right)$ form resonance states near the bottom of the conduction band, while $p$ - dopants (e.g., $\mathrm{Ga}\left(4 s^{2} 4 p^{1}\right)$, In $\left(5 s^{2} 5 p^{1}\right)$, and $\left.\mathrm{Tl}\left(6 s^{2} 6 p^{1}\right)\right)$ form resonance states at the top of the valence band. An increase of the DOS near $\varepsilon_{\mathrm{F}}$ has also been suggested based on experimental data for PbTe:Tl. ${ }^{[8,31]}$ Hence, a small increase of the DOS in TAGS-85 doped with Dy $\left(4 f^{10} 6 s^{2}\right)$ cannot be excluded, but, due to the low Dy concentration, the effect from the DOS modification cannot be large, and other mechanisms enhancing the thermopower should be considered.

It is possible that the enhancement of the thermopower observed in TAGS-85 doped with Dy is due to an energyfiltering mechanism (i.e., differential scattering of high- and low-energy carriers of the same sign by potential barriers in the material). ${ }^{[9-12]}$ The Seebeck effect requires that high-energy carriers diffuse from the hot end toward the cold end of the material and produce an electric field, which stops this motion and can be measured in the experiment. Low-energy carriers diffuse from the cold end toward the hot end, and the thermopower depends on the difference in the chemical potentials of the carriers with high and low energy. It is favorable if carriers with higher energy, moving from the hot end toward the cold end, are scattered less than those with lower energy moving from the cold end toward the hot end, ${ }^{[32,33]}$ resulting in a smaller cancellation of the two contributions and an enhancement of the thermopower.

Generally, the scattering can be associated with the parameters of the potential barriers in the material. In the energy filtering model, potential barriers may occur at the interfaces between the material matrix and nanoscale inclusions, ${ }^{[1,12]}$ or at grain boundaries due to Te vacancies. ${ }^{[11]}$ For TAGS-85 doped with Dy, we suggest that potential barriers can occur due to the mismatched atomic sizes and the large magnetic moment of the Dy atoms present in the lattice. A similar effect was discussed by Zide et al. ${ }^{[34]}$ for $\operatorname{In}_{0.53} \mathrm{Ga}_{0.47} \mathrm{As}$ with $\mathrm{In}_{0.53} \mathrm{Ga}_{0.28} \mathrm{Al}_{0.19} \mathrm{As}$ barriers. An effect due to $\mathrm{Mn}$ atoms with localized magnetic moments was observed in $\mathrm{Pb}_{0.16} \mathrm{Sn}_{0.72} \mathrm{Mn}_{0.12}$ Te by Radchenko et al. ${ }^{[35]}$

The electrical resistivity in semiconductors depends on the carrier concentration and mobility, which can be changed by doping. The most important mechanism affecting mobility at high temperatures is scattering by phonons. In paramagnetic materials, an additional contribution, magnetic scattering by localized magnetic moments, may increase the electrical resistivity. A large contribution may also arise from microcracks and the porosity, which are typical for multicomponent tellurides, and may overcome the contribution from the concentration and the mobility. Our data show that the enhancement of the power factor of TAGS-85 due to Dy doping was mostly achieved by the increase in the thermopower. Because the electrical resistivity of TAGS-85 doped with $2 \%$ Dy was larger than that in the sample doped with $1 \%$ Dy, its power factor was smaller.

It is well known that the thermal conductivity contains two contributions, due to the lattice and carriers, and is one of three components that affects the thermoelectric figure of merit. At $700 \mathrm{~K}$, the reported thermal conductivity of TAGS-85 is approximately $15 \mathrm{~mW} \mathrm{~cm}^{-1} \mathrm{~K}^{-1[26,27]}$ or $17 \mathrm{~mW} \mathrm{~cm}^{-1} \mathrm{~K}^{-1},{ }^{[28]}$ whereas our measurements showed $16 \mathrm{~mW} \mathrm{~cm}{ }^{-1} \mathrm{~K}^{-1}$. The effect of the thermal conductivity on $Z T$ is significant, but the uncertainty of the thermal transport measurements is large, $\geq 6 \%$. We expect that, due to the observed reduction in the carrier concentration and the distortion of the lattice induced by the doping of TAGS-85 with Dy, the thermal conductivity should also be reduced. This was confirmed by the data for TAGS-85 doped with $2 \%$ Dy, where the reduction in the thermal conductivity was clearly observed and can be attributed to an increase in the anharmonicity of the lattice vibrations (phonons), reducing heat propagation through the lattice. ${ }^{[36]}$ Again, the reported values of the power factor and figure of merit of TAGS-85 vary significantly. ${ }^{[18,26-28]}$ Our data, obtained for two samples, suggest that the ZT of TAGS-85 should be considered as $\leq 1.3$ at $730 \mathrm{~K}$ (as also indicated in the review by Snyder and Toberer ${ }^{[2]}$ ), and future studies should use this value as a reference. In TAGS-85 doped with Dy, the interplay between the electrical and thermal transport due to variable contributions from the carrier concentration, phonon scattering, and energy filtering results in an enhancement of $Z T$. 


\section{Conclusions}

TAGS-85 $\left(\mathrm{Ag}_{6.52} \mathrm{Sb}_{6.52} \mathrm{Ge}_{36.96} \mathrm{Te}_{50}\right)$, one of the best $p$-type thermoelectric materials, was doped with the rare earth dysprosium (Dy). Due to their large atomic size and high magnetic moment, Dy atoms may form selective barriers for electronic and thermal transport and enhance the thermoelectric efficiency. X-ray-diffraction, ${ }^{125} \mathrm{Te}-\mathrm{NMR}$ spectroscopy, and magnetic-susceptibility measurements of TAGS-85 doped with Dy (i.e., $\mathrm{Ag}_{6.52} \mathrm{Sb}_{6.52} \mathrm{Ge}_{35.96} \mathrm{Te}_{50} \mathrm{Dy}_{1}(1 \%$ Dy for Ge), $\mathrm{Ag}_{6.52} \mathrm{Sb}_{6.52} \mathrm{Ge}_{34.96} \mathrm{Te}_{50} \mathrm{Dy}_{2}$ (2\% Dy for Ge), and $\mathrm{Ag}_{6.52} \mathrm{Sb}_{6.52} \mathrm{Ge}_{36.96} \mathrm{Te}_{49} \mathrm{Dy}_{1}$ (1\% Dy for Te)) showed that some Dy atoms are incorporated into the lattice, where they produce large local lattice distortions. Doping of TAGS-85 with 1 or $2 \%$ Dy for Ge enhanced the thermopower by $\approx 7$ or $10 \%$, respectively, over a wide temperature range, from 305 to $730 \mathrm{~K}$; the power factor for TAGS-85 doped with 1\% Dy was enhanced by $\approx 25 \%$ compared with that in TAGS-85. The effect of doping on the thermopower, with Dy replacing Ge, was slightly stronger than that for Dy replacing Te. The enhancement of the Seebeck coefficient and power factor of TAGS-85, due to doping with Dy, was generally similar to that observed for doping with two other rare earths, $\mathrm{Ce}$ and $\mathrm{Yb}$. However, in contrast to Ce and $\mathrm{Yb}$, the 4 -electrons of Dy cannot form resonance states near the Fermi level. We suggest that the enhancement of the thermopower in TAGS-85 doped with Dy can be attributed partially to a reduction in the carrier concentration, detected by ${ }^{125} \mathrm{Te}$ NMR spectroscopy, and partially to energy filtering of the carriers by potential barriers associated with the Dy atoms, which, due to their large atomic size and localized magnetic moment, distort the TAGS-85 lattice. The interplay between the parameters of electronic and thermal transport in TAGS-85 due to doping with Dy resulted in the enhancement of ZT at $730 \mathrm{~K}$, from $\leq 1.3$ in TAGS- 85 to $\geq 1.5$ for TAGS- 85 doped with 1 or $2 \%$ Dy for Ge. Doping TAGS-85 with Dy forms a dilute magnetic semiconductor and makes it a promising material for thermoelectric power generation.

\section{Experimental Section}

Materials Synthesis and Sample Preparation: The TAGS-85 ingots doped with Dy were prepared by direct reaction of the constituent elements, $\mathrm{Ag}, \mathrm{Sb}, \mathrm{Ge}, \mathrm{Te}$, and $\mathrm{Dy}$, in fused silica ampoules. The initial nominal compositions were $\mathrm{Ag}_{6.52} \mathrm{Sb}_{6.52} \mathrm{Ge}_{35.96} \mathrm{Te}_{50} \mathrm{Dy}$ (TAGS-85 + 1\% Dy for Ge), $\mathrm{Ag}_{6.52} \mathrm{Sb}_{6.52} \mathrm{Ce}_{34.96} \mathrm{Te}_{50} \mathrm{Dy}_{2}$ (TACS-85 + 2\% Dy for Ge), and, in addition, $\mathrm{Ag}_{6.52} \mathrm{Sb}_{6.52} \mathrm{Ce}_{36.96} \mathrm{Te}_{49} \mathrm{Dy}$ (TAGS-85 + 1\% Dy for Te). The ampoules were heated up to $1323 \mathrm{~K}$ to melt the constituents and shaken periodically to form homogeneous ingots upon solidification. Finally, the melts were cooled down with the furnace. The master ingots had a mass of $20 \mathrm{~g}$, a diameter of $10 \mathrm{~mm}$, and a length of $\approx 36 \mathrm{~mm}$; measurements were made on several samples cut from each ingot. Several disc samples with a diameter of $\approx 10 \mathrm{~mm}$ and a thickness of $\approx 2 \mathrm{~mm}$ were prepared by cutting the ingot with a diamond saw perpendicular to the ingot axis. This allowed us to measure the variation of the Seebeck coefficient and electrical resistivity along the ingot as well as to use the same sample for different measurements. Two flat, parallel surfaces on opposite, initially curved sides of each disc sample were prepared for the thermopower and electrical resistivity measurements. The disc samples could also be used for thermal diffusivity measurements by the flash method. Small, bulk pieces of $\approx 0.2 \mathrm{~g}$ extracted from the disc samples were used for the magnetization measurements. For the X-ray-diffraction and
${ }^{125}$ Te-NMR-spectroscopy measurements, powder samples were prepared from the disc samples.

$X$-Ray Diffraction: Room temperature X-ray diffraction patterns were obtained for ground powders using a Scintag SDS-2000 diffractometer with $\mathrm{Cu} \mathrm{K}_{\alpha}$ radiation. The X-ray diffraction patterns obtained for the as-solidified TACS-85 + 1\% Dy for Te, TACS-85 + 1\% Dy for Ge, and TACS- $85+2$ at\% Dy for Ge were indexed to the rhombohedral $R 3 \mathrm{~m}$ polymorph (space group 160), which is a slight distortion of the hightemperature cubic structure of GeTe. TAGS- $x$ materials are known to exhibit a second-order polymorphic transformation from the hightemperature cubic to the low-temperature rhombohedral $R 3 m$ structure over a temperature range from 300 to $510 \mathrm{~K} \cdot{ }^{[21]}$

NMR Spectroscopy: Solid-state ${ }^{125} \mathrm{Te}-\mathrm{NMR}$-spectroscopy experiments were run on a Bruker Biospin (Billerica, MA) DSX-400 spectrometer (magnetic field of $9.3900 \mathrm{~T}$ ) at $126 \mathrm{MHz}$, using a $2.5 \mathrm{~mm}$ magicangle-spinning (MAS) probe head at a $22 \mathrm{kHz}$ spinning frequency; the sample masses were $\approx 30 \mathrm{mg} .{ }^{[20]}$ The signals were detected after a Hahn echo generated by a $2 \mu \mathrm{s}-t_{\mathrm{r}}-3.8 \mu \mathrm{s}-t_{\mathrm{r}}$ two-pulse sequence, where $t_{\mathrm{r}}$ denotes a rotation period. The second pulse and receiver phase were cycled according to the EXORCYCLE scheme. ${ }^{[3]}$ The measuring times were around $10 \mathrm{~h}$ for each spectrum. The ${ }^{125}$ Te-NMR-spectroscopy chemical shifts were referenced to $\mathrm{Te}(\mathrm{OH})_{6}$ in solution via solid $\mathrm{TeO}_{2}$ at $+750 \mathrm{ppm}$ as a secondary reference. The spin-lattice relaxation time, $T_{1}$, was determined by fitting the dependence of the normalized integral vs. the time delay after saturation by a train of $90^{\circ}$ pulses; ${ }^{[38]}$ the uncertainty of the $T_{1}$ determination was $\pm 0.2 \mathrm{~ms}$. Chemical-shift/dipolar anisotropy 125 Te-NMR-spectroscopy measurements ${ }^{[23]}$ were conducted to obtain information about the local structure of the materials studied. The unpaired electrons of the magnetic ions produced orientationdependent magnetic fields at the nuclei, which were probed by the NMR spectroscopy. The orientation-dependent (anisotropic) local fields were different for different crystallites and therefore led to destructive interference in the time signal, which manifested itself as a shortened relaxation time, $T_{2}{ }^{*}$ aniso. In addition, there were other factors shortening $T_{2}{ }^{*}$, such as a distribution of isotropic (orientation-independent) chemical or Knight shifts, resulting in $T_{2}{ }^{*}$ iso. In order to distinguish the anisotropic and isotropic fields, we needed MAS, which selectively refocused the anisotropic dephasing and thus increased $T_{2}{ }^{*}$ aniso without affecting $T_{2}{ }^{*}$ iso. In order to measure $T_{2}^{*}$ aniso, we applied a radio-frequency $\pi$-pulse that interfered with the MAS and, at the same time, refocused the decay due to $T_{2}^{*}$ iso ("Hahn spin-echo"). We used a simple pulse sequence, usually applied to analyze large chemical-shift anisotropies, with an excitation pulse, $t_{\mathrm{csa}}-\pi$-pulse- $t_{\mathrm{csa}}$, followed by an incremented " $z$-filter" (" $\gamma$-average") to remove spectral artifacts. ${ }^{[23]}$

Magnetization: The bulk dc magnetization of the samples was measured at 1.8 and $300 \mathrm{~K}$ using a Quantum Design superconducting quantum-interference device magnetometer in a magnetic field, $H$, varying from 0 to $55 \mathrm{kOe}$. The temperature dependence of the magnetic susceptibility was measured in the temperature range of $1.8-350 \mathrm{~K}$ in a $50 \mathrm{kOe}$ magnetic field. For the measurements, the samples were placed in a gel capsule of low diamagnetic susceptibility, $\chi=-1.3 \times 10^{-8}$ emu g ${ }^{-1} \mathrm{Oe}^{-1}$, an order of magnitude smaller than the lowest magnetic susceptibility of the samples. The uncertainties of the magnetic measurements were less than $2 \% .{ }^{[22]}$

Seebeck Coefficient (Thermopower) and Electrical Resistivity: Measurements of the Seebeck coefficient (thermopower) and electrical resistivity were performed using an LSR-3 measuring system (Linseis Inc.) in a helium atmosphere in the temperature range from 305 to $760 \mathrm{~K}$. The Seebeck coefficient and the electrical resistivity were measured simultaneously for the same sample using a four-probe method; the temperature difference for thermopower measurements was between 8 and $9 \mathrm{~K}$ in the entire temperature range, with an uncertainty of $\leq 0.1 \mathrm{~K}$. The Seebeck coefficient was measured relative to the Pt legs of a $\mathrm{Pt}-(\mathrm{Pt}+\mathrm{Rh})$ thermocouple and then the absolute Seebeck coefficient was calculated. The uncertainties of the thermopower and electrical resistivity measurements were 5 and $3 \%$, respectively.

Comments on the Seebeck Coefficient Measurements: It is worth noting here that important experimental details must be taken into consideration 
to obtain meaningful data, in particular if rather small changes of the physical parameter are of importance. Firstly, in measurements, the relative Seebeck coefficient is obtained, while, as a characteristic of a material, the absolute Seebeck coefficient is needed. ${ }^{[39]}$ At $700 \mathrm{~K}$, the relative Seebeck coefficient of $p$-type TAGS-85 material measured with Pt probes is $201 \mu \mathrm{V} \mathrm{K}^{-1}$, whereas the absolute Seebeck coefficient is $188 \mu \mathrm{V} \mathrm{K}^{-1}$ (i.e., smaller by $\approx 13 \mu \mathrm{V} \mathrm{K}{ }^{-1}$ ). ${ }^{[40]}$ If the relative value instead of the absolute Seebeck coefficient were used to calculate the power factor of a $p$-type material, its value could be artificially larger by $\approx 15 \%$. Secondly, Lowhorn et al. ${ }^{[4]]}$ reported that measurements of samples of similar composition prepared in 12 different laboratories, or even of the same samples, may show significantly different thermopower values; a similar situation was also reported by D'Angelo et al. ${ }^{[42]}$ Hence, measurements of reference materials and then several samples of the initial and modified materials conducted under the same conditions are needed to reduce the uncertainty of measurements and assess the effect of doping. For reference, we measured $n$-type $\mathrm{Ni}_{80} \mathrm{Cr}_{20}$, and its absolute thermopower agreed well with published data. ${ }^{[41,42]}$ In addition, in order to understand the homogeneity of the doped material, we studied the variation of the thermopower along the ingot.

Thermal Diffusivity and Conductivity: The thermal diffusivity, $\lambda$, and the specific heat, $c_{\mathrm{p}}$, were measured using an XFA-500 measuring system (Linseis Inc.) in a helium atmosphere by the flash method; the uncertainty of the measurements was $6 \%$. The thermal conductivity, $\kappa$, was calculated as $\kappa=\lambda c_{\mathrm{p}} d$, where $d$ is the mass density of the material.

\section{Acknowledgements}

The authors wish to thank L. P. Lincoln at the Materials Preparation Center at Ames Laboratory, US Department of Energy, for sample synthesis. S.L.B. and E.M.L. thank P. C. Canfield (ISU) for his interest in this work. This work was supported by the US Department of Energy, Office of Basic Energy Sciences, Division of Materials Sciences and Engineering. The research was performed at the Ames Laboratory, which is operated for the US Department of Energy by lowa State University under Contract No DE-AC02-07CH11358.

Received: December 15, 2011 Published online: April 10, 2012

[1] M. S. Dresselhaus, G. Chen, M. Y. Tang, R. Yang, H. Lee, D. Wang, Z. Ren, J.-P. Fleurial, P. Gogna, Adv. Mater. 2007, 19, 1043.

[2] G. J. Snyder, E. S. Toberer, Nat. Mater. 2008, 7, 105.

[3] Y. Lan, A. J. Minnich, G. Chen, Z. Ren, Adv. Funct. Mater. 2010, 20, 357.

[4] D. Kraemer, B. Poudel, H.-P. Feng, C. Caylor, B. Yu, X. Yan, Y. Ma, X. Wang, D. Wang, A. Muto, K. McEnaney, M. Chiesa, Z. Ren, G. Chen, Nat. Mater. 2011, 10, 532

[5] J. Karni, Nat. Mater. 2011, 10, 481.

[6] Y. Pei, X. Shi, A. LaLonde, H. Wang, L. Chen, G. J. Snyder, Nature 2011, 473, 66

[7] J. P. Heremans, C. M. Trush, D. T. Morelli, J. Appl. Phys. 2005, 98, 063703.

[8] J. P. Heremans, V. Jovovic, E. S. Toberer, A. Saramat, K. Kurosaki, A. Charoenphakdee, S. Yamanaka, G. J. Snyder, Science 2008, 321, 554.

[9] Y. I. Ravich, in CRC Handbook of Thermoelectrics (Ed: D. M. Rowe) CRC Press LLC, Boca Raton, FL 1995, Ch. 7.

[10] Y. Nisho, T. Hirano, Jpn. J. Appl. Phys. 1997, 36, 170.

[11] K. Kishimoto, M. Tsukamoto, T. Koyanagi, J. Appl. Phys. 2001, 92, 65331.
[12] S. V. Faleev, F. Leonard, Phys. Rev. B: Condens. Matter 2008, 77, 214304.

[13] S. Chikazumi, Physics of Ferromagnetism, $2^{\text {nd }}$ ed. Oxford University Press, New York 1997.

[14] N. B. Brandt, V. V. Moshchalkov, Adv. Phys. 1984, 33, 373.

[15] Periodic table of the elements with their atomic radii, http://www. standnes.no/chemix/periodictable/atomic-radius-elements.htm (accessed March 2012)

[16] K. Uchida, S. Takahashi, K. Harii, J. leda, W. Koshibae, K. Ando, S. Maekawa, E. Saitoh, Nature 2008, 455, 778.

[17] C. M. Jaworski, J. Yang, S. Mack, D. D. Aweschalom, J. P. Heremans, R. C. Myers, Nat. Mater. 2010, 9, 898.

[18] E. A. Skrabek, D. S. Trimmer, in CRC Handbook of Thermoelectrics (Ed: D. M. Rowe) CRC Press LLC, Boca Raton, FL 1995, Ch. 22.

[19] C. Woods, Rep. Prog. Phys. 1988, 51, 459

[20] E. M. Levin, B. A. Cook, J. L. Harringa, S. L. Budko, R. Venkatasubramanian, K. Schmidt-Rohr, Adv. Funct. Mater. 2011, 21, 441.

[21] B. A. Cook, M. J. Kramer, X. Wei, J. L. Harringa, E. M. Levin, J. Appl. Phys. 2007, 101, 53715.

[22] E. M. Levin, X. W. Fang, S. L. Bud'ko, W. E. Straszheim, R. W. McCallum, K. Schmidt-Rohr, Phys. Rev. B: Condens. Matter 2008, 77, 054418.

[23] J.-D. Mao, K. Schmidt-Rohr, Solid State Nucl. Magn. Reson. 2004, 26, 36.

[24] S. Gorsse, P. B. Pereira, R. Decourt, E. Sellier, Chem. Mater. 2010, 22, 988.

[25] S. P. Li, J. Q. Li, Q. B. Wang, L. Wang, F. S. Liu, W. Q. Ao, Solid State Sci. 2011, 13, 399.

[26] S. H. Yang, T. J. Zhu, T. Sun, J. He, S. N. Zhang, X. B. Zhao, Nanotechnology 2008, 19, 245707.

[27] J. R. Salvador, J. Yang, X. Shi, H. Wang, A. A. Wereszczak, J. Solid State Chem. 2009, 182, 2088.

[28] E. A. Skrabek, D. Trimmer, United States Patent \#3945855, 1976.

[29] G. A. Slack, S. Galginaitis, Phys. Rev. 1964, 133, A253.

[30] S. Ahmad, K. Hoang, S. D. Mahanti, Phys. Rev. B: Condens. Matter 2006, 74, 155205.

[31] Y. Matsushita, H. Bluhm, T. H. Geballe, I. R. Fisher, Phys. Rev. Lett. 2005, 94, 157002.

[32] M. Horvat, T. Prosen, G. Casati, Phys. Rev. B: Condens. Matter 2009, $80,010102(R)$

[33] G. Benenti, G. Casati, Philos. Trans. R. Soc. London A 2011, 369, 466.

[34] J. M. O. Zide, D. Vashaee, Z. X. Bian, G. Zeng, J. E. Bowers, A. Shakouri, A. C. Gossard, Phys. Rev. B: Condens. Matter 2006, 74, 205335.

[35] M. V. Radchenko, G. V. Lashkarev, V. Osinniy, B. Witkowska, V. Domukhovski, T. Story, J. Magn. Magn. Mater. 2002, 247, 77.

[36] J. E. Turney, E. S. Landry, A. J. H. McGaughey, C. H. Amon, Phys. Rev. B: Condens. Matter 2009, 79, 064301.

[37] G. Bodenhausen, R. Freeman, D. L. Turner, J. Magn. Reson. 1977, 27, 511.

[38] E. M. Levin, B. A. Cook, K. Ahn, M. G. Kanatzidis, K. Schmidt-Rohr, Phys. Rev. B: Condens. Matter 2009, 80, 115211.

[39] J. Martin, T. Tritt, C. Uher, J. Appl. Phys. 2010, 108, 121101.

[40] A. T. Burkov, in Thermoelectrics Handbook, Macro and Nano (Ed: D. M. Rowe) CRC Press, Boca Raton, FL 2006, Ch. 22.

[41] N. D. Lowhorn, W. Wong-Ng, W. Zhang, Z. Q. Lu, M. Otani, E. Thomas, M. Green, T. N. Tran, N. Dilley, S. Ghamaty, N. Elsner, T. Hogan, A. D. Downey, Q. Jie, Q. Li, H. Obara, J. Sharp, C. Caylor, R. Venkatasubramanian, R. Willigan, J. Yang, J. Martin, G. Nolas, B. Edwards, T. Tritt, Appl. Phys. A - Mater. Sci. Process. 2009, 94, 231.

[42] J. D’Angelo, A. Downey, T. Hogan, Rev. Sci. Instrum. 2010, 81, 075107. 